FOLIA

Amazónica

Revista del Instituto de Investigaciones

de la Amazonía Peruana

\title{
EL ELA TRADICIONAL: EL USO DE LAS ESPECIES VEGETALES DE LOS HUMEDALES EN EL TEJIDO DEL PUEBLO URARINA EN LA CUENCA DEL RÍO CHAMBIRA, LORETO, PERÚ
}

\author{
Manuel MARTÍN BRAÑAS ${ }^{1}$, Margarita DEL AGUILA VILLACORTA ${ }^{1}$,

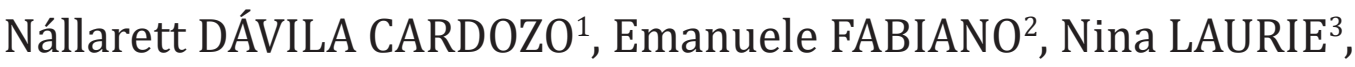 \\ Wendy MOZOMBITE RUÍZ ${ }^{1}$, Juan José PALACIOS VEGA ${ }^{1}$, \\ Cecilia del Carmen NÚÑEZ PÉREZ ${ }^{1}$
}

1 Instituto de Investigaciones de la Amazonía Peruana - IIAP. Av. Abelardo Quiñones km 2,5. Iquitos, Perú. Correo electrónico: mmartin@iiap.gob.pe

2 Pontificia Universidad Católica del Perú - PUCP. Av. Universitaria 1801. Lima, Perú.

3 Universidad de Saint Andrews. College Gate Saint Andrews KY16 9AJ. Scotland, United Kingdom.

\section{RESUMEN}

En la cuenca del río Chambira, Loreto, Perú, las mujeres Uranina son conocidas por tejer esteras llamadas "ela" o "cachihuango" de la fibra de la palmera aguaje (Mauritia flexuosa L.f.) y otras especies de los humedales. El tejido "ela" se convierte en seña de identidad que une el pasado con el presente de las nuevas generaciones, a través del vínculo entre la maestra tejedora y la joven aprendiza. El objetivo de este estudio fue identificar las especies vegetales utilizadas por dos comunidades del pueblo urarina en sus tejidos tradicionales. Aplicamos entrevistas semi-estructuradas, observamos las prácticas textiles y visitamos las zonas donde las especies de plantas fueron colectadas. Se determinó el uso, directo o indirecto, de siete especies vegetales utilizadas en el tejido tradicional "ela". Los hallazgos refuerzan la idea de la adaptación tardía del pueblo urarina a los humedales y la importancia que la práctica tradicional tiene para la conservación de los ecosistemas inundables.

PALABRASCLAVE: Fibras vegetales, tintes vegetales, uso tradicional, ecosistemas inundables. 


\title{
THE TRADITIONAL ELA: THE URARINA'S TRADITIONAL USE OF PLANT SPECIES FROM WETLANDS FOR WEAVING IN THE CHAMBIRA RIVER WATERSHED, LORETO, PERU
}

\begin{abstract}
In the Chambira River Watershed, Loreto, Peru. Uranina women are well known for weaving mats called ela or cachihuango from aguaje palm (Mauritia flexuosa) fiber and other wetland plant species. The ela fabric becomes a sign of identity that unites past and present generations, through the link made between the elder master weaver and the young apprentice. The objectives of this study were to explore the plant species used by two urarinas communities in the production of mats. We conducted semi-structured interviews, observed textile practice, and visited areas where plant species were collected. We identified seven plant species that were used directly or indirectly in the production of ela. The findings reinforce the idea of the late adaptation of the Urarina people to wetlands and the potential role that traditional practice has for its conservation.
\end{abstract}

KEY WORDS: Vegetal fibers, vegetal dyes, traditional use, flood ecosystems. 


\section{INTRODUCCIÓN}

Los tejidos elaborados con las fibras de diferentes especies vegetales evidencian la adaptación de los pueblos indígenas amazónicos a un medio natural adverso para el ser humano. La interpretación del entorno natural les ha permitido desarrollar habilidades y conocimientos que han favorecido su supervivencia y desarrollo permanente. El profundo conocimiento de las especies aprovechadas, transmitido por generaciones, favorece la elaboración de objetos tejidos que han sido usados en diferentes ámbitos de su vida cotidiana y espiritual.

Pero los tejidos amazónicos no deben ser entendidos como una simple habilidad artesanal, ya que incorporan conocimientos integrales que van más allá de las tramas tejidas, convirtiéndose, a menudo, en el puente que une el pasado ancestral con el presente de los mayores y el futuro de las nuevas generaciones.

El tejido tradicional "ela" del pueblo urarina, cuya lengua aislada es hablada por 4000 a 6000 personas en el departamento de Loreto, Perú (Dean, 1994; Walker, 2013), es el ejemplo más evidente del pensamiento integral indígena, ya que se convierte en el eje sobre el que gira su universo cultural. Para elaborar los tejidos, las mujeres usan un tipo de telar de cintura prehispánico, un préstamo tecnológico cuyo origen no es claro, pero que bien podría provenir de los pueblos amazónicos del Ecuador, quienes posiblemente la adoptaron de los pueblos andinos; o de los pueblos de ascendencia jíbaro que habitaban los territorios adyacentes y que habrían difundido la práctica (Dole, 1998; Martín et al., 2019). Algunas de las técnicas de teñido utilizadas por el pueblo urarina son similares a las usadas por pueblos como los quijo, que habitaban cerca de la actual frontera con Perú, en los territorios que hoy pertenecen al Ecuador (Oberem, 1980).
Las mujeres del pueblo urarina utilizan diferentes especies vegetales para la elaboración de sus tejidos tradicionales. El tejido, conocido localmente con el nombre de "cachihuango", término que proviene de las palabras en lengua quechua "cachi" - sal—y "wanku” - paño, lienzo, envoltorio de tela- (Martín et al., 2019), y en lengua urarina con el nombre "ela", se caracteriza por la yuxtaposición paralela de hilos o grupos de hilos vegetales de diferentes colores. Es utilizado como estera de descanso y es un objeto personal que acompaña al hombre y a la mujer durante toda su vida, por lo que juega un papel fundamental en el día a día de las comunidades urarina. Hay que recordar que la ausencia de hamacas para el descanso es una característica de pueblos de ascendencia jibaro, pero también lo era de pueblos como los mainas, los roamainas y los propios urarina (Renard-Casevitz, 1988). En Brasil y en Colombia, pueblos como el xavante, el guahivo, el apinaye o el mekranoti, usan de manera cotidiana esteras para el descanso nocturno (Smith, 2015).

El presente artículo expone los resultados del trabajo de identificación de las especies vegetales usadas por las maestras tejedoras de dos comunidades urarinas de la cuenca del río Chambira. Se determinan los usos dados a cada una de las especies vegetales y el papel que cumplen en la práctica tradicional. La identificación de las especies vegetales permitirá establecer estrategias para su manejo sostenible, así como iniciar programas de revalorización de los conocimientos tradicionales basados en la puesta en valor de los objetos tejidos con alto valor cultural agregado.

\section{MATERIAL Y MÉTODOS}

\section{ÁREA DE ESTUDIO}

El área de estudio se localizó en las comunidades de Nueva Unión y Nueva Pandora, ubicadas 
Figura 1: Mapa de ubicación geográfica de las comunidades Nueva Unión y Nueva Pandora, Loreto, Perú.

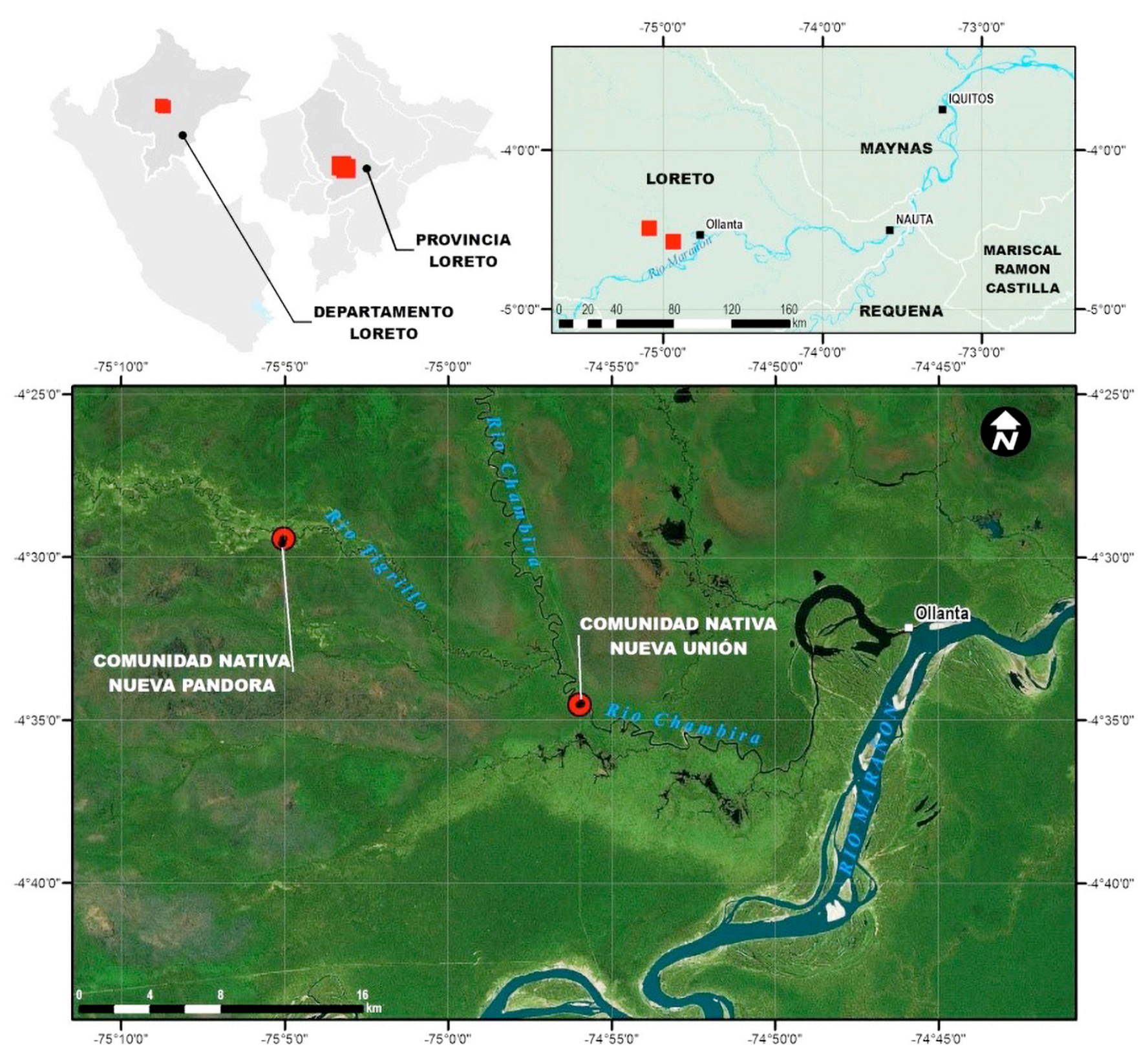

en las cuencas de los ríos Chambira y Tigrillo respectivamente, este último, afluente del río Chambira. Las comunidades pertenecen al distrito Urarinas, provincia de Loreto, departamento de Loreto, Perú, ubicándose en las siguientes coordenadas geográficas $74^{\circ} 55^{\prime} 58.02^{\prime \prime}$ Oeste $4^{\circ} 34^{\prime} 32.34 "$ Sur y $75^{\circ}$ 5'1.54" Oeste - 4²9'26.62" Sur, respectivamente (Figura 1). El acceso a estas comunidades es por vía terrestre desde la ciudad de Iquitos hasta la ciudad de Nauta, tomando un tiempo de una hora con 30 minutos, luego, por vía fluvial desde Nauta al centro poblado Ollanta, tomando de siete a ocho horas en un motor de 60 caballos de fuerza; de Ollanta hacia Nueva Unión se accede por vía fluvial surcando el río Chambira, tomando una hora si se utiliza el mismo motor; 
finalmente de Nueva Unión a Nueva Pandora se accede también por vía fluvial en una hora y 45 minutos aproximadamente. Según el último censo de población y vivienda del año 2017 del Instituto Nacional de Estadística e Informática (INEI), la comunidad Nueva Unión está conformada por una población de 101 habitantes; mientras que Nueva Pandora por doce habitantes. La investigación se llevó a cabo durante los años 2017 al 2019, implicando estadías periódicas de más de dos semanas de duración en las comunidades. La investigación cumplió con los estándares éticos de investigación establecidos por el comité de ética de la Universidad de Saint Andrews y las leyes de consulta previa vigentes en el Perú.

\section{LEVANTAMIENTO DE INFORMACIÓN Y COLECTA DE MUESTRAS}

Se presentan los datos sistematizados de las entrevistas semi estructuradas aplicadas en las dos comunidades para registrar los conocimientos sobre las especies vegetales vinculadas con la práctica del tejido tradicional y los usos dados a cada una de ellas. Las entrevistas fueron complementadas con información etnográfica recogida en talleres participativos desarrollados en cadauna delascomunidadesyacompañamiento en las actividades de colecta, preparación de los materiales y tejido de las tramas. Previo a los talleres se solicitó el consentimiento previo informado, tal como marca la Ley $\mathrm{N}^{\circ} 27811$.

Se realizó un trabajo de colecta (Tabla 1) de las especies usadas en la práctica del tejido, identificadas previamente en los talleres comunales y en las encuestas semi estructuradas. La colecta se realizó con el acompañamiento de las maestras tejedoras de ambas comunidades, después de identificar las especies de uso en campo, estas fueron colectadas usando tijera telescópica y herborizadas de acuerdo a lo recomentado por Judd et al. (1999). Las muestras se depositaron en el Herbario Herrerense ( $\mathrm{HH})$ del Instituto de Investigaciones de la Amazonía Peruana. La identificación de las especies fue realizada con guías de identificación (Vásquez, 1997; Ribeiro et al., 1999) y por comparación en el Herbario

Tabla 1: Datos de colecta de las especies utilizadas para la elaboración del tejido ela.

\begin{tabular}{|c|c|c|c|c|c|c|c|c|}
\hline Código & Familia & Especie & Hábito & $\begin{array}{c}\text { Altura } \\
\text { (m) }\end{array}$ & $\begin{array}{c}\text { Este } \\
\text { (m) }\end{array}$ & Norte (m) & $\begin{array}{l}\text { Zona } \\
\text { UTM }\end{array}$ & Fecha \\
\hline $\begin{array}{l}\text { Observación } \\
\text { en campo }\end{array}$ & Arecaceae & $\begin{array}{l}\text { Mauritia } \\
\text { flexuosa }\end{array}$ & Palmera & --- & --- & --- & --- & --- \\
\hline $\begin{array}{l}\text { Observación } \\
\text { en campo }\end{array}$ & Arecaceae & $\begin{array}{c}\text { Oenocarpus } \\
\text { mapora }\end{array}$ & Palmera & --- & --- & --- & --- & --- \\
\hline $\begin{array}{l}\text { Observación } \\
\text { en campo }\end{array}$ & Arecaceae & $\begin{array}{l}\text { Iriartea } \\
\text { deltoidea }\end{array}$ & Palmera & --- & --- & --- & --- & --- \\
\hline Dávila 7098 & Bignoniaceae & $\begin{array}{c}\text { Fridericia } \\
\text { chica }\end{array}$ & Hierba & 4 & 490813 & 9503608 & 18 Sur & 2/10/2019 \\
\hline Dávila 7099 & Rubiaceae & $\begin{array}{l}\text { Simira } \\
\text { rubescens }\end{array}$ & Árbol & 3,5 & 490770 & 9503650 & 18 Sur & 3/10/2019 \\
\hline Dávila 7100 & Combretaceae & $\begin{array}{c}\text { Buchenavia } \\
\text { viridiflora }\end{array}$ & Árbol & 3 & 490678 & 9503581 & 18 Sur & 3/10/2019 \\
\hline Dávila 7101 & Combretaceae & $\begin{array}{l}\text { Terminalia } \\
\text { dichotoma }\end{array}$ & Árbol & 10 & 490962 & 9503688 & & 26/11/2019 \\
\hline
\end{tabular}


Herrerense. Los nombres fueron verificados usando la base de datos TROPICOS del Jardín Botánico de Missouri (http://www.tropicos.org). El permiso de colecta de las muestras de plantas corresponde a la Resolución Gerencial Regional: 0041-2019-GRL-GRDFFS.

\section{RESULTADOS Y DISCUSIÓN}

Las mujeres del pueblo urarina utilizan, de manera directa o indirecta, un total de siete especies vegetales en la práctica del tejido tradicional, tanto para realizar las tramas como para preparar los tintes que embellecen los tejidos: Buchenavia viridiflora Ducke y Terminalia dichotoma G.Mey (Combretaceae), Fridericia chica (Bonpl.) L.G. Lohmann (Bignoniaceae), Iriartea deltoidea Ruiz \& Pav., Mauritia flexuosa L.f. y Oenocarpus mapora H.Karst (Arecaceae) y Simira rubescens (Benth.) Bremek. ex Steyerm. (Rubiaceae) (Tabla 2).

\section{EL TEJIDO URARINA}

La especie que cobra más importancia en el tejido es el aguaje ("ala" - Mauritia flexuosa), usada tanto para dar forma a la urdimbre, como para desarrollar la trama misma del tejido (Dean, 2009; Martín et al., 2019).
Mauritia flexuosa es una especie emblemática de la Amazonía. Es una palmera grande, con un único tallo columnar que puede alcanzar una altura de $35 \mathrm{~m}$ y un diámetro a la altura del pecho (DAP) de $60 \mathrm{~cm}$. Sus hojas son costapalmadas y su fruto se caracteriza por estar cubierto de escamas de color marrón rojizo-anaranjado a rojo oscuro. Se distribuye en Bolivia, Brasil, Colombia, Ecuador, Guayana Francesa, Trinidad y Tobago, Guyana, Perú y Venezuela.

Las mujeres urarina obtienen la fibra base para el tejido de las hojas jóvenes o velas ("alaa alu") de la palmera de $M$. flexuosa que todavía no despliegan sus foliolos. Por lo general, son aprovechadas las palmeras juveniles, cuyos brotes pueden ser cosechados sin mayor dificultad, debido a que la palmera no ha alcanzado mucha altura. El proceso para obtener la fibra de $M$. flexuosa es muy similar al realizado para obtener la fibra de la chambira ("risine" - Astrocaryum chambira Burret, Arecaceae), utilizada ampliamente en toda la Amazonía (Vormisto, 2002; García et al., 2015; Smith, 2015).

El uso de la fibra de $M$. flexuosa en el tejido es una particularidad del pueblo urarina que fue informada inicialmente por los primeros misioneros que llegaron a sus territorios (Jiménez, 1898; Figueroa, 1904; Renard-Casevitz,

Tabla 2: Especies vegetales y partes usadas en el tejido urarina.

\begin{tabular}{|c|c|c|c|c|}
\hline Especie & $\begin{array}{l}\text { Nombre } \\
\text { común }\end{array}$ & $\begin{array}{l}\text { Nombre } \\
\text { urarina }\end{array}$ & $\begin{array}{l}\text { Parte } \\
\text { usada }\end{array}$ & Uso \\
\hline Mauritia flexuosa & Aguaje & Ala & $\mathrm{Hj} ; \mathrm{V} ; \mathrm{Ef}$. & Fibra para urdimbre y trama del tejido. \\
\hline Fridericia chica & Pucapanga & Lüriane & $H$. & Tintura (rojo) para la fibra. \\
\hline Simira rubescens & Pucarito & Anuriaa & Cv. & Corteza que se mezcla con lüriane. \\
\hline Buchenavia viridiflora & Yacushapana & Uruijia & $\mathrm{Cm}$. & Corteza que se mezcla con lüriane. \\
\hline Terminalia dichotoma & Yacushapana & Uruijia & $\mathrm{Cm}$. & Corteza que se mezcla con lüriane. \\
\hline Iriartea deltoidea & Huacrapona & Eechu & R. & $\begin{array}{c}\text { Rallador para lüriane. Material lanzador } \\
\text { bichu nüjüa. }\end{array}$ \\
\hline Oenocarpus mapora & Sinamillo & Ünaee & Hj; Ef. & Material cernidor. \\
\hline
\end{tabular}


1988). Si bien, hay otros pueblos, como por ejemplo el yagua, que actualmente utilizan las fibras extraídas de las hojas jóvenes de $M$. flexuosa (Chaumeil, 2002), estos pueblos no utilizan el telar de cintura para elaborar tejidos complejos con la fibra de esta palmera. La presencia en sus territorios de grandes extensiones de "aguajales", conocidos por el pueblo urarina como "alaka" (Schulz et al., 2019), es un factor importante para entender el uso cotidiano de la fibra en la práctica del tejido. Se estima que en el espacio delimitado de norte a sur por la cabecera del río Chambira y el río Ucayali, y de este a oeste por los ríos Pastaza y Tigre, existen más de dos millones de hectáreas de aguajales (Draper et al., 2014). No cabe duda, que la disponibilidad de este recurso se convierte en el principal condicionante para su uso. Por otro lado, los urarinas usan los tejidos "ela" como esteras para el descanso y la fibra extraída de $M$. flexuosa es mucho más suave y confortable que la de otras especies vegetales, como por ejemplo la chambira, siendo su fibra idónea para la elaboración de este tipo de esteras.

Para la extracción de la fibra, las mujeres o los hombres urarinas cortan los brotes de la palmera con un machete y los transportan hasta sus casas. Las mujeres sacuden los brotes en el suelo para separar los foliolos. Una vez que los foliolos están visibles, quiebran sus puntas y separan las fibras no vasculares adheridas a la epidermis del foliolo. Para eliminar el color verdoso de las fibras naturales y blanquearlas, las mujeres las hierven en agua durante media hora, colocándolas posteriormente al sol durante dos o tres días. Pasado el proceso de blanqueado, las fibras se pueden teñir o se procede inmediatamente al proceso de torcido (Martín et al., 2019).

El torcido de la fibra de $M$. flexuosa es el proceso por el cual se obtienen los hilos con los que se elaborarán los tejidos tradicionales. Para ello, utilizan una técnica particular en la que involucran tanto los pies como las manos. Las mujeres fijan las fibras entre el primer dedo del pie o hallux y el segundo dedo del pie, con las manos tensan las fibras y las giran en un mismo sentido, logrando que todas se entrelacen. Para la urdimbre se tuercen dos hebras y para la trama se tuercen tres. Las fibras torcidas se acomodan como urdimbre en el telar o se enrollan en el lanzador o bobina ("bichu nüjüa"), fabricado con la madera de la huacrapona ("eechu" - Iriartea deltoidea), elemento que permitirá intercalar las tramas. Es común encontrar en la casa de las maestras tejedoras madejas de fibra de diferentes colores que están convenientemente preparadas para ser utilizadas en el tejido.

\section{TIÑENDO LAS FIBRAS DE AGUAJE}

Las mujeres urarina alternan hilos teñidos con tintes vegetales, con hilos de color natural, lo que hace a los tejidos mucho más vistosos. Para teñir las fibras, las mujeres urarinas utilizan varias especies vegetales, algunas de uso tradicional, las cuales describimos a continuación.

La principal especie vegetal que utilizan para dar color a las fibras es la pucapanga ("lüriane" Fridericia chica), un arbusto o liana de hasta tres metros, con hojas compuestas y opuestas, con inflorescencia paniculada y flores tubulares, la corola lila a púrpura y los frutos son cápsulas de 3,8 x 1,5 cm. Esta especie es de amplia distribución en toda América del Sur. En Perú se la encuentra en tierra firme en bosque transicional y también como planta cultivada (Vásquez, 1997).

Las tejedoras urarina obtienen de esta especie un color rojizo oscuro (lanaji) típico de sus tejidos. La savia de Fridericia chica, al ser expuesta al ambiente, adquiere un color rojizo característico, debido a la presencia de antocianinas y carotenoides como la genipina, la bixina y varios derivados de la carajurina (Vega, 2001; Amaral et al., 2012), siendo aprovechada como recurso tintóreo por muchos pueblos indígenas. 
Las mujeres urarina rallan las hojas de Fridericia chica con un rallador fabricado con la raíz de la palmera huacrapona ("eechu" - Iriartea deltoidea), las hojas ralladas las mezclan con agua. La masa resultante es colada en un cernidor fabricado con los foliolos jóvenes del sinamillo ("ünaee"- Oenocarpus mapora H.Karst.). La fibra es sumergida en el líquido resultante durante un día. Al terminar el proceso, la fibra adquiere un color rojo característico que corresponde al código 10R3/10 del sistema de clasificación de colores de Munsell (Munsell, 1915; Espinoza, 2016).

El color extraído de la hoja de Fridericia chica puede ser modificado si se mezcla con otras especies vegetales o con arcilla. Para obtener un color negro azulado, a la fibra teñida con la hoja de F. chica se la entierra durante medio día en un tipo de arcilla de color marrón claro que se encuentra en las orillas de las quebradas. La arcilla, debido a su acidez y a su alto contenido de metales (Riveros \& Inga, 2014), actúa como mordiente del tinte, fijándolo y modificando el color rojizo a un color negro azulado.

Para conseguir un color rojo brillante con menor intensidad, las mujeres utilizan las cortezas de las especies vegetales pucaquiro ("anuriaa" - Simira rubescens) y yacushapana ("uruijia" - Buchenavia viridiflora y Terminalia dichotoma), estas últimas especies, conocidas con el mismo nombre común, presentan carotenoides esenciales que proporcionan un color rojizo característico (Riveros \& Castillo, 2014). Simira rubescens es un árbol pequeño o mediano de hasta $10 \mathrm{~m}$ de altura; su tronco es recto y cilíndrico (Williams, 1936), las hojas son simples y opuestas, con inflorescencia globosa y flores tubulares, el fruto es tipo cápsula subgloboso de 2,5-4 x 3-4,5 cm. Se distribuye en Brasil, Colombia y Perú (Taylor et al., 2004 ). Buchenavia viridiflora es un árbol que alcanza los $30 \mathrm{~m}$ de altura, con raíces tablares y una copa amplia; sus hojas son simples y alternas, su inflorescencia es tipo espiga, los frutos son tipo drupa oblongas de 15-20 x 8 mm. (Vásquez, 1997). Esta especie está distribuida en Brasil, Colombia, Guayana Francesa, Guyana y Perú. Por último, Terminalia dichotoma es un árbol que puede alcanzar los $30 \mathrm{~m}$ de altura, con hojas simples obovadas u oblongas, presenta una copa ovalada grande y densa (Lorenzi, 2009), la inflorescencia es tipo espiga y los frutos tipo drupas obovoides (Vasquez, 1997). Ocurre en Brazil, Colombia, Ecuador, Guyana, Perú y Venezuela.

La corteza muerta, tanto de la especie Buchenavia viridiflora, como de la especie Terminalia dichotoma se hierven junto a las hojas de Fridericia chica. En una olla colocan los trozos de corteza y las hojas ralladas, agregando un poco de agua y dejando cocinar por dos horas aproximadamente, revolviendo de vez en cuando. Pasado el tiempo de cocción, se deja enfriar en la misma olla. Para separar los restos vegetales del agua, se filtra todo con la ayuda de un colador o paño de trama fina. El líquido resultante adopta un color rojo intenso, más claro y brillante que el color aislado de F. chica. En este líquido sumergen las fibras de Mauritia flexuosa durante dos días. Las fibras teñidas no son colocadas al sol para su secado, debido a que perderían el color brillante.

La corteza viva de la especie Simiira rubescens es raspada y mezclada con agua en una olla inmediatamente después de extraerla, sumergiendo la fibra en ella durante uno o dos días, hasta que adquiera el color rosado brillante. El resultado para cualquiera de las especies, una vez hervida junto con Fridericia chica, es un tinte que adquiere un color que se corresponde con el código 10R3/8 del sistema de clasificación de Munsell (Munsell, 1915; Espinoza, 2016).

Los colores usados para teñir las fibras que darán forma al tejido tradicional "ela" son de suma importancia para el pueblo urarina, siendo algo más que un simple recurso estético, ya que tienen una relación muy importante con la espiritualidad 
urarina y representan la vida y la salud familiar (Martin et al., 2019).

\section{LA FIBRA DE Mauritia flexuosa EN LA AMAZONÍA}

Los pueblos indígenas amazónicos siempre han usado las especies vegetales de su entorno de manera sostenible (Dufour, 1990). Un buen número de investigaciones etnobotánicas evidencian la importancia que tiene el uso de las especies vegetales para las comunidades amazónicas (Boom, 1989; Pinedo, 1990; Galeano, 2000; Shanley \& Rosa, 2004; Marín et al., 2005; Cadena et al., 2007; Trujillo \& Correa, 2010; Rengifo et al., 2017; Pyhälä, 2003).

El alto porcentaje de uso de las especies vegetales, es un indicador claro de la efectiva adaptación del ser humano al medio amazónico. El poblador amazónico ha interpretado su entorno natural, aumentando de manera progresiva su conocimiento sobre las especies vegetales y animales, incorporando estrategias culturales que le han permitido interactuar de manera equilibrada y segura en ecosistemas tan especiales y sumamente adversos para el ser humano (Morán, 1991). El largo proceso de adaptación dibujó en toda la Amazonía un mapa de especialización que encajaba en el rompecabezas de la diversidad ecosistémica. La especialización trajo consigo un uso particular de las especies vegetales, atendiendo a varios factores, como por ejemplo, su disponibilidad o abundancia en cada uno de los ecosistemas.

El uso de la fibra de Mauritia flexuosa, materia prima fundamental para los tejidos del pueblo urarina, fue informado por primera vez en las crónicas de los misioneros jesuitas y franciscanos que llegaron a la Amazonía y la Orinoquía. Cristóbal de Saavedra, miembro de la expedición encabezada por el gobernador Diego Vaca de Vega a los territorios del pueblo maina, reportó en 1620, el uso que este pueblo daba a las fibras de esta familia de plantas (Renard-Casevitz et al., 1988). En su relación del viaje, señala la importancia de estos tejidos, informando incluso de los trueques habituales realizados con ellos (Jiménez, 1898). En 1661 el Padre Francisco de Figueroa, en su informe sobre las misiones jesuitas en el río Marañón, realiza una breve descripción de los lienzos tejidos por el pueblo roamaina con las fibras de $M$. flexuosa, señalando que es más fácil de labrar, pero de menor duración que el algodón (Figueroa, 1904 [1661]). Siete décadas después, en 1736, el padre jesuita Pablo Maroni relata el uso que los indígenas dan a las fibras de $M$. flexuosa para la elaboración de "telas listadas muy curiosas, conocidas como cachibancos" (Maroni, 1988 [1738]). En la Orinoquía, el padre jesuita Joseph Gumilla, reportó en 1745, el uso que el pueblo Guarauno daba a las fibras de la palma de M. flexuosa para fabricar esteras para el descanso, ropa y soguillas. (Gumilla, 1745). A las puertas del siglo XIX, el padre Francisco Veigl hace también referencia al uso de la fibra de $M$. flexuosa por los pueblos urarina y maina, utilizada para confeccionar telas "conocidas como cachibancos" (Veigl, 2006 [1798]).

Varios han sido los investigadores que han reportado el uso contemporáneo de la fibra de Mauritia flexuosa. Balick (1979), señala como los indios guahivo de Colombia usan la fibra de las hojas jóvenes como complemento decorativo de las hamacas tejidas con Astrocaryum chambira. El mismo autor informa sobre el uso que le dan los indios apinayé del noreste brasileño a la fibra de $M$. flexuosa para la elaboración de canastas (Balick, 1988). En el Perú, los yagua del bajo Amazonas y Napo, usan las fibras de las hojas jóvenes de $M$. flexuosa para elaborar sus prendas tradicionales (Chaumeil, 1987; Smith, 2015). Los mekranoti, que viven en los territorios bañados por el río Xingu, Iriri y Curuá, en Brasil, fabrican, al igual que los xavante, esteras con su fibra (Verswijver, 1996). El mismo autor reporta cómo 
los kayapó de los ríos Xingú y Tocantins fabrican bolsas, adornos, canguros y cinturones con la fibra de $M$. flexuosa. Coimbra et al. (2002) señala como las mujeres xavante extraen las fibras de sus hojas jóvenes para fabricar esteras para el descanso. Los xavante utilizan la misma técnica de torcido que las mujeres urarinas, pero no utilizan el telar de cintura, ya que el tejido lo realizan a mano de manera vertical. Los pueblos xinguano, del alto río Xingu, utilizan la fibra de las hojas de $M$. flexuosa para fabricar cuerdas y esteras (Heckenberger, 2005). Las mujeres nukak y las piapoco de Colombia tejen hamacas con la fibra de la epidermis de la hoja de M. flexuosa (Mesa \& Galeano, 2013; Smith, 2015). Los kichwa del alto Pastaza en Ecuador, utilizan sus fibras como cortinas en sus viviendas (Smith, 2015).

\section{EL TELAR URARINA: ADAPTACIÓN Y ESPECIALIZACIÓN A LOS ECOSISTEMAS INUNDABLES}

Si bien, el uso de la fibra de Mauritia flexuosa, como hemos visto, ha estado bastante extendido en toda la Amazonía, no ha sido tan habitual el uso del telar de cintura para tejerla. Existen referencias sobre el uso de la fibra de $M$. flexuosa en telares de madera con bastidores verticales. Los pueblos yekuana y yanomani de Venezuela elaboran hamacas y vestimentas con diferentes fibras vegetales, incluida la fibra del $M$. flexuosa, utilizando un telar de bastidores verticales (Delgado, 2013). Abboud (1975), informa sobre el uso que pueblos como el jíbaro, el piro, el omagua y el cocama, todos del Amazonas peruano, dan a los telares de cintura para tejer con las fibras del algodón. Los amahuaca del Perú, tejen sus hamacas de algodón en telares verticales y sus prendas de vestir, también de algodón, en telares de cintura (Dole, 1998). Asimismo, los pueblos awajun y shipibo en el Perú (Taish, 2001; Wali,
2016) y shuar en el Ecuador (Bianchi, 1982) han utilizado o utilizan tanto el telar vertical simple como el telar de cintura para tejer con algodón sus vestimentas tradicionales. Ninguno de estos autores reporta el uso de la fibra de M. flexuosa en telares de cintura.

El tejido de la fibra de Mauritia flexuosa en el telar de cintura es una particularidad que refuerza la teoría de la especialización del pueblo urarina a un ecosistema inundable donde prolifera esta palmera. Asimismo, las especies Buchenavia viridiflora y Simira rubescens, utilizadas para teñir las fibras de M. flexuosa, son especies características de los aguajales y las zonas inundables. Es muy probable que esta especialización haya sido tardía, ya que el pueblo urarina, antes de ubicarse en los actuales territorios, probablemente habitó las cabeceras de los ríos Tigre, Pastaza y Morona, cerca de la actual frontera con Ecuador (Dole, 1998; Martín et al., 2019). No hay datos que nos permitan aventurar las causas de esta migración, pero probablemente se llevó a cabo debido a las presiones de otros pueblos que compartían esos territorios, entre los que posiblemente estaban algunos de ascendencia jíbaro (Dean, 1999) o kandozi y otros de origen pano que migraron progresivamente hacia el sur (Dole, 1998; Olawsky, 2006). No obstante, a la llegada de los primeros cronistas españoles a sus territorios, los mismos que ocupan actualmente, el tejido con la fibra de $M$. flexuosa ya estaba totalmente extendido (Figueroa, 1904 [1661]; Veigl, 2006 [1798]). En su antigua ubicación es posible que usaran el algodón como materia prima en sus telares, de la misma forma que hoy lo usan los pueblos jíbaro que habitan en estos territorios. Al moverse a los territorios inundables que ocupan actualmente, la escasez de la planta de algodón forzó una adaptación de los tejidos a la nueva realidad. 


\section{PLANTAS TINTÓREAS EN LAS ÁREAS INUNDABLES DEL PUEBLO URARINA}

El uso de diferentes especies vegetales para la elaboración de tintes ha sido una práctica muy extendida en la Amazonía. La especie Fridericia chica ha sido muy importante para las culturas de la América tropical (Roquero, 2008). Su amplia distribución en todo el continente ha permitido que varias culturas amazónicas originarias la usen habitualmente (Groome, 1998; Tessman, 1999; Ocampo \& Balick, 2009; Smith, 2015). En el Perú, la mayoría de pueblos indígenas amazónicos la conocen y la usan habitualmente para sus pinturas corporales o para teñir fibras (Tessman, 1999; Polesna et al., 2009; Odonne et al., 2013).

No abundan los reportes sobre Simira rubescens para elaborar tintes. Antúnez (1989) registra el uso de la corteza de S. tinctoria para elaborar tintes de color rojizo. Williams (1936), informa sobre el color rosa oscuro que toma la albura de S. tinctoria al contacto con el aire, pero no reporta su uso para elaborar tintes. Delprete \& Nee (1997), reportan como las comunidades del Valle del río Huallaga usan la corteza de la especie Simira macrocater K. Schum (Rubiaceae) para teñir ropa y fibras de la palmera chambira Astrocaryum chambira. García et al. (2015) reporta el uso que el pueblo ticuna de la Amazonía colombiana da a la especie Simira cordifolia Hook f. (Rubiaceae), como tinte de color rojo para teñir la fibra de la chambira.

El uso de las especies de los géneros Buchenavia y Terminalia también ha sido reportado. Polesna et al. (2009) reporta el uso que da el pueblo shipibo conibo en el Perú a la corteza de Buchenavia oxycarpa (Mart.) Eichler (Combretaceae) para la elaboración de tintes de color marrón. Riveros \& Inga (2014), Riveros \& Castillo (2014) y Gonzáles (2018), reportan el uso de la especie Buchenavia parvifolia Ducke (Combretaceae) para la elaboración de tintes. Shabbir et al. (2016), reporta la obtención de tintes de la especie Terminalia chebula Retz (Combretaceae). Sinha et al. (2016) reporta el uso de Terminalia arjuna (Roxb.) Wight \& Arn. (Combretaceae) para la obtención de tintes de color amarillo marrón. Tournon et al. (2015) reporta el uso que le da el pueblo shipibo conibo del Perú a la especie Terminalia oblonga (Ruiz y Pav.) Steud. (Combretaceae) para teñir tejidos.

No se han encontrado reportes sobre tintes obtenidos a partir de la combinación de Fridericia chica con las especies arbóreas Simira rubescens, Buchenavia viridiflora o Terminalia dichotoma. El color obtenido con la combinación de estas especies es igual al color obtenido con la especie Bixa orellana L. (Bixaceae) (Espinoza, 2016), abundante en los suelos de altura, pero casi inexistente en los suelos inundables. Una investigación más profunda podría determinar si la combinación fue originalmente planteada por el pueblo urarina como una alternativa para conseguir el color obtenido con la especie $B$. orellana, sobre todo en aquellas comunidades que habitan en suelos expuestos a la inundación periódica y donde $B$. orellana no es tan frecuente.

\section{CONCLUSIÓN}

La identificación de siete especies vegetales utilizadas por el pueblo urarina para la elaboración de sus tejidos tradicionales "ela", es solo una muestra más de la profundidad e integralidad del conocimiento indígena amazónico. Las especies utilizadas como materia prima para la obtención defibray tintes que son utilizados en la elaboración del "ela", son características de los aguajales y los bosques inundables, evidenciándose una especialización de las comunidades de estudio a este tipo de hábitats. Tanto las técnicas utilizadas en la práctica del tejido, como el mismo telar de cintura, muestran una clara conexión con la de otros pueblos indígenas que habitan más al norte. Estos son elementos que refuerzan la idea de la 
migración y la adaptación tardía a este tipo de ecosistemas. El uso de las especies vegetales de las zonas inundables en los tejidos tradicionales se convierte en su principal garantía de conservación. Mientras el tejido "ela" mantenga su estatus central en la cultura urarina, los bosques que albergan las especies utilizadas para su elaboración serán respetados y conservados, siendo de todas formas necesaria la incorporación de prácticas de manejo sostenible que minimicen el impacto, tanto del uso tradicional, como del potencial uso comercial. La práctica del tejido y el uso sostenible de las especies vegetales no será posible sin el mantenimiento de la identidad y los valores propios del pueblo urarina.

\section{AGRADECIMIENTOS}

Este estudio no hubiera sido posible sin el financiamiento de "Cienciactiva / CONCYTEC", British Council, La Embajada Británica, y NewtonPaulet Fund mediante el Contrato-№2202018-FONDECYT, correspondiente al proyecto "Protecting Biodiversity and livelihoods in the wetlands of peruvian Amazonia", así como por los fondos de la Universidad de St Andrews, Escocia, recibidos a través del Proyecto "Gaining cultural heritage status for urarina textiles: A pathway to peatland livelihoods and ecological conservation in Loreto, Perú".

\section{REFERENCIAS BIBLIOGRÁFICAS}

Abboud, R. 1975. South American Backstrap Loom: Its Potential, Limitation and Application for Contemporary Handweaving. Tesis de maestría. University of Rhode Island, Rhode Island, EEUU. 231pp.

Amaral, R.; Santos, A.; Saravia, A.; Botas, G.; Cruz, R.; Fernándes, C.; Rocha, L.; Boylan, F.
2012. Biological activities of Arrabidaea chica (Bonpl.) B. Verl. leaves. Latin American Journal of Pharmacy, 31(3): 451.

Antúnez, K. 1989. Peruvian natural dye plants. Economic Botany, 43(2): 189.

Balick, M. 1979. Economic botany of the Guahibo. I. Palmae. Economic Botany, 33(4): 364. DOI: https://doi.org/10.1007/BF02858332

Balick, M. 1988. The use of palms by the Apinayé and Guajajara Indians of Northeastern Brazil. In: Balick, M.J. (Ed). The palm, tree of life: biology, utilization and conservation. Advances in Economic Botany, 6: 65-90.

Bianchi, C. 1982. Artesanías y técnicas shuar. Mundo Shuar, Quito. 477pp.

Boom, B.M. 1989. Use of Plant Resources by the Chácobos. Resource Management in Amazonia: Indigenous and Folk Strategies. Advances Economic Botany, 7: 78-96.

Cadena, C.; Diazgranados, M.; Bernal, H. 2007. Plantas útiles para la elaboración de artesanías de la comunidad indígena monifue amena (Amazonas-Colombia). Universitas Scientarum, 12(1): 97-116.

Chaumeil, J.P. 1987. Ñinamwo los yagua del nororiente peruano. Centro Amazónico de Antropología y Aplicación Práctica, Lima.

Chaumeil, J.P. 2002. The blowpipe indians. In: Rival, L.M.; Whitehead, N. L (Eds). Beyond the Visible and the Material: The Amerindianization of Society in the Work of Peter Rivière, p. 81-99. Coimbra, C.; Flowers, N.; Salzano, F.; Santos, R. 2002. The Xavante in transition: health, ecology, and bioanthropology in central Brazil. University of Michigan Press, Ann Arbor. 376pp.

Dean, B. 1994. The poetics of creation: Urarina cosmogony and historical consciousness. Latin American Indian Literature Journal, 10(1): 2245.

Dean, B. 1999. Intercambios ambivalentes en la Amazonia: formación discursiva y la violencia 
del patronazgo. Anthropologica, 17(17): 85115.

Dean, B, 2009. Urarina society, cosmology and history in Peruvian Amazonia. University Press of Florida, Florida. 344pp.

Delgado, L. 2013. Huellas de lo sagrado: estetismo y grafismo de lo sagrado. Ministerio del poder popular para la cultura, Caracas.

Delprete, P.; Nee, M. 1997. The enigmatic genus Wernhamia S. Moore is a synonym of Simira (Rubiaceae, Rondeletieae). Brittonia, 49(3): 303-308.

Dole, G. 1998. Los Amahuaca. In: Santos, F.; Barclay, F. (Eds.), Guía etnográfica de la Alta Amazonía. Volumen III. Lima: IFEA, Smithsonian Tropical Research Institute, Abya Yala, p 125-274.

Draper, F.; Roucoux, K.; Lawson, I.; Mitchard, E.; Honorio, E.; Lähteeenoja, O.; Torres L.; Valderrama, E.; Zárate, R.; Baker, T. 2014. The distribution and amount of carbon in the largest peatland complex in Amazonia. Environmental Research Letters, 9(12): 2. DOI: http://dx.doi. org/10.1088/1748-9326/9/12/124017

Dufour, D. 1990. Use of tropical rain forests by native Amazonia. Bioscience, 40(9): 652-659.

Espinoza, G. 2016. Tintes vegetales de la sierra y selva del Perú: Un estudio etnobotánico en los departamentos de Ancash, Loreto y Cusco. Tesis de grado. Universidad Peruana Cayetano Heredia, Lima, Perú. 89pp.

Figueroa, F. 1904 [1661]. Relación de las misiones de la Compañía de Jesús en el país de los Maynas. Librería General de Victoriano Suárez, Madrid.

Galeano, G. 2000. Forest use at the Pacific Coast of Chocó. Economic Botany, 54: 358.376.

García, N.; Galeano, G.; Mesa, L.; Castaño, N.; Balslev, H.; Bernal, R. 2015. Management of the palm Astrocaryum chambira Burret (Arecaceae) in northwest Amazon. Acta Botanica Brasilica, 29(1). DOI: http://dx.doi.org/10.1590/0102$33062014 a b b 3415$
Gonzáles, I. 2018. Identificación de productos forestales no maderales - Tintes vegetales en la zona Intag, Noroccidente del Ecuador. Tesis de pre-grado. Universidad Técnica del Norte, Ibarra, Ecuador. 67 p.

Groome, S. 1998. Arrabidaea chica (Bignoniaceae): An ethnobotanical study of its biology, domestication potencial and uses by two groups, the Bribri and the Cabécar of Costa Rica. San José, Costa Rica: ACM Tropical Field Research.30 p.

Gumilla, J. 1745. El Orinoco ilustrado y defendido. Historia natural, civil y geographica de este gran rio: govierno, usos y costumbres de los indios... / escrita por el padre Joseph Gumilla, de la Compañia de Jesús... Manuel Fernández, Madrid. 407pp.

Heckenberger, M.J. 2005. The ecology of power: culture, place, and personhood in the southern Amazon, A.D. 1000-2000. Routledge, New York. 432pp.

Jiménez, M. (comp.). 1898. Relaciones geográficas de indias: Perú. Volumen 4. Ministerio de Fomento, Madrid. 312pp.

Judd, W.; Campbell, C.; Kellogg, E.; Stevens, P. 1999. Plant Systematics A phylogenetic approach. Sinauer Associates, Inc. Sunderland, Reino Unido. 464pp.

Lorenzi, H. 2009. Brazilian trees. Volume 3. Instituto Plantarum, Nova Odessa, Brasil.

Marín, C.; Cárdenas, D.; Suárez, S. 2005. Utilidad del valor de uso en etnobotánica. Estudio en el departamento de Putumayo (Colombia). Caldasia, 27(1): 89-101.

Maroni, P S.J.1988 [1738]. Noticias auténticas del famoso río Marañón y misión apostólica de la Compañía de Jesús. Centro de Estudios Teológicos de la Amazonía, Colección Monumenta Amazónica, Iquitos, Perú. 565pp.

Martín, M.; Núñez, C.; Fabiano, E.; Del Aguila, M.; Schulz, C.; Laurie, N.; Sanjurjo, J.; Davies, A.; Roucoux, K.; Lawson, I.; Andueza, L. 2019. Urarina: identidad y memoria en la cuenca del 
río Chambira. MINAM, IIAP, PUCP, Universidad de Saint Andrews, Iquitos, Perú. 76pp.

Mesa, L.; Galeano, G. 2013. Usos de las palmas en la Amazonía colombiana. Caldasia, 35(2): 351369.

Morán, E. 1991. Human Adaptive Strategies in Amazonian Blackwater Ecosystems. American Anthropologist. 93(2):361-382. DOI: https:// doi.org/10.1525/aa.1991.93.2.02a00050

Munsell A. H. 1915. Atlas of Munsell Color System. Malden, Mass., Wadsworth, Howland \& Co., inc., Printers.

Oberem, U. 1980. Los quijos. Historia de la transculturación de un grupo indígena en el Oriente Ecuatoriano. Instituto Otavaleño de Antropología, Otavalo, Ecuador. 394pp.

Ocampo, R.; Balick, M. 2009. Plants of semillas sagradas: an ethnomedicinal garden in Costa Rica. Finca Luna Nueva Extractos de Costa Rica. Odonne, G.; Valadeau, C.; Albán, J.; Stien, D.; Sauvain, M.; Bourdy, G. 2013. Medical ethnobotany of the chayahuita of the Paranapura basin (Peruvian Amazon). Journal of Ethnopharmacoly, 146: 127:153. DOI: https://doi.org/10.1016/j. jep.2012.12.014

Olawsky, K. 2006. A grammar of urarina. Mouton de Gruyter, Berlín, Alemania. 943pp.

Pinedo, M.; Zarin, D; Jipp, P.; Chota, J. 1990. Usevalues of tree species in a communal forest reserve in northeast Peru. Conservation Biology, 4(4): 405-416. DOI: https://doi. org/10.1111/j.1523-1739.1990.tb00315.x

Polesna, L.; Polesny, Z.; Lachmanova, I.; Clavo, M.; Kokoska, L. 2009. Ethnobotanical study of dye yielding plants used in communities of shipibokonibo Amerindians around Pucallpa city, Perú. In: Rojas, N; Prieto, R. (Eds.), The Amazon basin: Plant life, Wildlife and Environment. Nova science publishers, p 123-141.

Pyhälä, A. 2003. Productive Conservation in Amazonia: Institutions, participation and markets. Tesis de grado. University of East Anglia, U.K. 306pp.

Renard-Casevitz, F.M.; Saignes, Th.; Taylos, A.C. 1988. Al este de los Andes: Relaciones entre las sociedades amazónicas y andinas entre los siglos XV y XVII. Abya Yala, Quito. 329pp.

Rengifo, E.; Ríos, S.; Fachín, L.; Vargas, G. 2017. Saberes ancestrales sobre el uso de flora y fauna en la comunidad indígena Tikuna de Cushillo Cocha, zona fronteriza Perú-Colombia-Brasil. Revista Peruana de Biología, 24(1): 67-78. DOI: http://dx.doi.org/10.15381/rpb.v24i1.13108

Ribeiro, J. E. L. S.; Hopkins, M.J. G.; Vicentini, A; Sothers, C. A.; Costa, M. A. S.; Brito, J. M.; Souza, M.A.D.; Martins, L.H.; Lohmann, L. G.; Assunção, P.A.; Pereira, E. C.; Silva, C. F.; Mesquita, M. R.; Procópio, L. C. 1999. Flora da Reserva Ducke. Guia de identificação das plantas vasculares de uma floresta de terra firme na Amazônia Central. INPA-DFID, Manaus. 800pp.

Riveros, L.; Inga, L. 2014. Caracterización química de los extractos colorantes de siete especies forestales y del fijador natural, utilizado en 19 comunidades indígenas de Ucayali, Perú. Ciencia Amazónica, 4(1): 29-36. DOI: https:// doi.org/10.22386/ca.v4i1.65

Riveros, L.; Castillo, A. 2014. Especies maderables y no maderables con sustancias colorantes utilizadas para el teñido y pintado de telas en 19 comunidades indígenas de la región Ucayali, Perú. Ciencia Amazónica, 4(1): 37-44. DOI: https://doi.org/10.22386/ca.v4i1.66

Roquero, A. 2008. Identification of Red Dyes in Textiles from the Andean Region. Textile Society of America Symposium Proceedings, 129.

Schulz, C.; Martín, M.; Núñez, C.; Del Aguila, M.; Laurie, N.; Lawson, I.; Roucoux, K. 2019. Peatland and wetland ecosystems in Peruvian Amazonia: indigenous classifications and perspectives. Ecology and Society, 24(2). DOI: https://doi.org/10.5751/ES-10886-240212 
Shabbir, M.; Islam, S.; Bukhari, M.; Rather, L.; Khan, M. 2016. Application of Terminalia chebula natural dye on wool fiber-evaluation of color and fastness properties. Textiles and Clothing Sustainability, 2(1). DOI: https://doi. org/10.1186/s40689-016-0011-8

Shanley, P.; Rosa, N. 2004. Eroding knowledge: an ethnobotanical inventory in Eastern Amazonia`s logging frontier. Economic Botany, 58. DOI: https://doi.org/10.1663/00130001(2004)058[0135:EKAEII]2.0.CO;2

Sinha, K.; Aikat, K.; Das, P.; Dattab, S. 2016. Dyeing of modified cotton fiber with natural Terminalia arjuna:optimization of dyeing parameters using response surface methodology. Enviromental Progress Sustainable Energy. 35(3): 719-728. DOI: https://doi.org/10.1002/ep.12284

Smith, N. 2015. Palms and people in the Amazon. Springer. 500pp.

Taish, J. 2001. Pedagogía ancestral awajún: la elaboración de textiles y su enseñanza en las comunidades de Nuevo Israel y Nuevo Jerusalén. Tesis de post grado. Universidad Mayor de San Simón, Cochabamba, Bolivia, p 73-78.

Taylor, C. M.; Steyermark, J. A.; Delprete, P. G.; Vincentini, A.; Cortés, R.; Zappi, D.; Persson, C.; Costa, C.B. \& Anunciação, E. 2004. Rubiaceae. In: Steyermark, J.A.; Berry, P.E.; Yatskievych, K.; Holst, B. K. (Eds.). Flora of the Venezuelan Guayana, 8: 497-847

Tessman, G.1999. Los indígenas del Perú nororiental. Abya Yala, Quito, Ecuador. 486pp.

Tournon, J.; Enocaise, F.; Caúper, S.; Cumapa, C.; Etene, C.; Panduro, G.; Riva, R.; Sánchez, J.; Tenazoa, M.; Urquia, R. 2015. Etnobotánica de los shipibo conibo. In: Horak, M. (Ed.). Etnobotánica y fitoterapia en América. Universidad de Mendel, Berno, República Checa, p 110-163.
Trujillo, W.; Correa, M. 2010. Plantas usadas por una comunidad indígena coreguaje en la Amazonía colombiana. Caldasia, 32(1): 1-20. DOI: http://dx.doi.org/10.15446/caldasia

Vásquez, R. 1997. Flórula de las Reservas Biológicas de Iquitos, Perú, AllpahuayoMishana, Explornapo Camp, Explorama Lodge. Monographs in Systematic Botany from the Missouri Botanical Garden, Vol. 63. St. Louis, MO. 1046pp.

Vega, M. 2001. Etnobotánica de la Amazonía peruana. Abya Yala, Quito, Ecuador. 166pp.

Veigl, F.C. 2006 [1798]. Noticias detalladas sobre el estado de la provincia de Maynas en América Meridional hasta el año de 1768. CETA, Iquitos, Perú. 261pp.

Verswijver, G. 1996. Mekranoti: living among the painted people of the Amazon. Prestel, Munich. 162pp.

Vormisto, J. 2002. Making and marketing chambira hammocks and bags in the Village of Brillo Nuevo, Northeastern Peru. Economic Botany, 56(1): 27-40. DOI: https://doi. org/10.1663/0013-0001(2002)056[0027:MA MCHA]2.0.CO;2

Wali, A.; Odland, J.; Belaunde, L. E.; Gardner, N.; Morales, D.; Mujica, A.; Weber, R. 2016. The Shipibo-Conibo: culture and collections in context. Fieldiana Anthropology, 45(1): 1-100. DOI: https://doi.org/10.3158/0071-473945.1.1

Walker, H. 2013. Wild Things: Manufacturing Desire in the Urarina Moral Economy. The Journal of Latin American and Caribbean Anthropology, 18(1): 51-66. DOI: https://doi. org/10.1111/j.1935-4940.2012.01254.x

Williams, L. 1936. Woods of northeastern Perú. Field Museum of Natural History. Botanical Series 15. 587pp.

Recibido: 22 de abril de 2020 Aceptado para publicación: 2 de junio de 2020 